\title{
CD9 regulates transcription factor GCM1 and ERVWE1 expression through the cAMP/protein kinase A signaling pathway
}

\author{
Yoshikage Muroi, Toshihiro Sakurai, Akira Hanashi, Kentaro Kubota, Kentaro Nagaoka and \\ Kazuhiko Imakawa \\ Laboratory of Animal Breeding, Graduate School of Agricultural and Life Sciences, The University of Tokyo, 1-1-1 \\ Yayoi, Bunkyo-ku, 113-8657 Tokyo, Japan \\ Correspondence should be addressed to K Imakawa; Email: akaz@mail.ecc.u-tokyo.ac.jp
}

Y Muroi is now at Laboratory of Veterinary Pharmacology, Obihiro University, 11 West 2, Inada-cho, Obihiro, Hokkaido 080-8555, Japan.

\begin{abstract}
ERVWE1 (SYNCYTIN-1), a membrane protein originating from the envelope gene of human endogenous retrovirus-W (HERV-W), mediates the fusion of mononucleated cytotrophoblasts into multinucleated syncytiotrophoblast. Though ERVWE1 has been characterized since its discovery, regulatory mechanisms associated with ERVWE1 expression have not been firmly established. We hypothesized that membrane protein CD9, involved in cell-cell fusion of fertilization and myogenesis, could be involved in the regulation of ERVWE1 gene expression. In this study, regulatory mechanisms of ERVWE1 expression were studied using human choriocarcinoma BeWo cells. Forskolin is an activator of adenylate cyclase, which increased CD9 and ERVWE1 expression. The increase in CD9 expression was inhibited by a protein kinase A (PKA) inhibitor, Rp-cAMPS. These results indicate that CD9 expression is regulated by the CAMP/PKA signaling pathway. Overexpression of CD9 increased expression levels of ERVWE1 as well as GCM1 (hGCMa), which is a transcription factor known to activate $E R V W E 1$ gene transcription. However, high ERVWE1 expression induced by $C D 9$ overexpression did not result in the increase in chorionic gonadotropin, beta polypeptide production. Moreover, CD9-induced increase in ERVWE1 and GCM1 expressions were inhibited by Rp-cAMPS. These results suggest that CD9 increases GCM1 expression via the cAMP/PKA signaling pathway, resulting in the increase in ERVWE1 expression.

Reproduction (2009) 138 945-951
\end{abstract}

\section{Introduction}

Trophoblasts are the first differentiated cells in embryonic development and play an important role in both embryonic implantation to the uterine endometrium and placental formation (Ferretti et al. 2007). Human trophoblasts are classified into two cell types; cytotrophoblasts and syncytiotrophoblasts. Cytotrophoblasts are mononucleated cells that proliferate and also form anchoring villi, from which these cells invade the maternal endometrium (Aplin 1991). Cytotrophoblasts also fuse and differentiate into multinucleated syncytiotrophoblasts (Midgley et al. 1963). Syncytiotrophoblasts exhibit numerous functions including the transportation of nutrients and gases and the synthesis of hormones such as placental lactogen, chorionic gonadotrophin, and progesterone, all of which are indispensable for pregnancy establishment and maintenance (Benirschke \& Kaufmann 2001). Therefore, these products have been used as markers of cell-cell fusion of trophoblast cells (Jameson \& Hollenberg 1993).
Although the molecular mechanism for placental morphogenesis has not yet been determined, it was demonstrated that SYNCYTINs, which are the membrane proteins derived from the envelope protein of endogenous retroviruses, play a key role in the fusion of cytotrophoblasts, resulting in the formation of syncytiotrophoblasts. Human SYNCYTINs are classified into two types, SYNCYTIN-1 (now called ERVWE1) and SYNCYTIN-2, which are derived from human endogenous retrovirus-W (HERV-W) and human endogenous retrovirus-FRD (HERV-FRD), respectively (Mi et al. 2000, Blaise et al. 2003). Both types of SYNCYTINs are fusogenic (Mi et al. 2000, Blaise et al. 2003, Malassiné et al. 2007) and are expressed in both cytotrophoblasts and syncytiotrophoblasts (Blond et al. 2000, Frendo et al. 2003, Malassiné et al. 2007). The effect of SYNCYTINs on the fusion of human cytotrophoblast cells has been well characterized. However, except cAMP/protein kinase $A(P K A)$ signaling pathway, its regulatory mechanism has not been definitively characterized. Activation of the cAMP signaling increases ERVWE1 
gene expression (Frendo et al. 2003, Chen et al. 2008) and can induce syncytia formation in primary cytotrophoblasts and human choriocarcinoma BeWo cells (Feinman et al. 1986, Mi et al. 2000). Activation of the CAMP/PKA signaling pathway in turn increases the expression of a transcription factor, glial cell missing factor homolog 1 (GCM1, previously called hGCMa), which binds to the upstream region of the ERVWE1 gene and then stimulates $E R V W E 1$ gene transcription in a cellspecific manner (Yu et al. 2002, Chang et al. 2005).

It is still unclear which molecules regulate the cAMP/PKA signaling pathway, resulting in the activation of ERVWE1 gene transcription. The members of the tetraspanin superfamily (transmembrane four superfamily; Maecker et al. 1997, Berditchevski 2001s) constitute the 'tetraspanin web', which is the complex molecular network which interacts with each tetraspanin and/or other membrane proteins (Boucheix \& Rubinstein 2001). CD9, a member of the tetraspanin superfamily, is required for cell-cell fusions in which fertilization takes place (Le Naour et al. 2000) and also in myogenesis (Tachibana \& Hemler 1999). In macrophages, CD9 regulates secretions of IL10 and IL6 via the CAMP/PKA signaling pathway (Ha et al. 2005). We hypothesized that CD9 regulates ERVWE1 gene expression through cAMP/PKA signaling pathway and a transcription factor GCM1 in human trophoblast cells. Therefore, the purpose of the present study was to examine whether or not the ERVWE1 gene is upregulated by CD9 in BeWo cells, and to identify molecular mechanisms by which the ERVWE1 gene is regulated by $\mathrm{CD} 9$.

\section{Results}

\section{CD9 expression is regulated by $C A M P / P K A$ signaling pathway}

To identify a factor(s) that was upregulated following the forskolin treatment in BeWo cells, several candidate mRNAs were examined. Among them, increase in $C D 9$ mRNA was found, which was subjected to further studies. During the course of such examinations, the effect of forskolin on ERVWE1 and chorionic gonadotropin, beta polypeptide (CGB) expression was examined. It was found that ERVWE1 and CGB expression levels in BeWo cells were increased after the forskolin treatment for 24, 48, or $72 \mathrm{~h}$ (Fig. 1A and B). CD9 mRNA and protein expression levels were also increased with the forskolin treatment for 24, 48, or $72 \mathrm{~h}$ (Fig. 2A and B). The increase in CD9 expression induced by forskolin was inhibited by $50 \mu \mathrm{M}$ Rp-cAMPS (Fig. 2C). These results indicate that CD9 could be regulated through the cAMP/PKA signaling pathway.
A
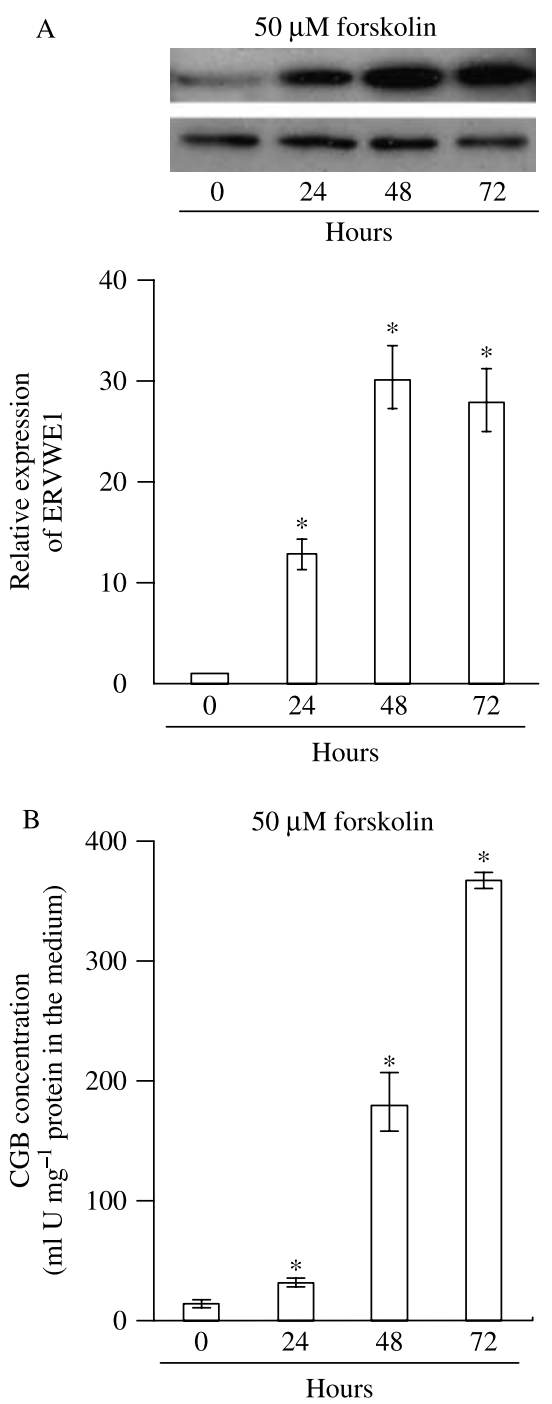

Figure 1 The effects of forskolin on ERVWE1 and CGB expression. (A) Western blot analysis of ERVWE1 and ACTB in BeWo cells treated with $50 \mu \mathrm{M}$ forskolin for $0,24,48$, or $72 \mathrm{~h}$. The data from three independent experiments were analyzed by ImageJ software (NIH). Expression levels of ERVWE1 normalized by ACTB are presented as mean \pm S.E.M. from three independent experiments. ${ }^{*} P<0.05$, compared with $0 \mathrm{~h}$. The inset shows the representative data of ERVWE1 (upper lane) and ACTB (lower lane). (B) ELISA of CGB in the medium in which cultured BeWo cells were treated with $50 \mu \mathrm{M}$ forskolin for 0,24 , 48 , or $72 \mathrm{~h}$. The data are presented as mean \pm s.E.M. from three independent experiments. ${ }^{*} P<0.05$, compared with $0 \mathrm{~h}$.

\section{CD9 up-regulates the expression of ERVWE1 via cAMP/PKA signaling pathway}

To delineate molecular mechanisms by which CD9 could possibly regulate ERVWE1 expression, its expression was evaluated following the transfection of CD9 expression plasmid into BeWo cells. Transient transfection of pSG5-CD9 demonstrated the increase in CD9 expression in BeWo cells (Fig. 3A). Transient transfection of pSG5-CD9 also increased the abundance of ERVWE1 and $C G B$ mRNAs in BeWo cells (Fig. 3B). Similar to 


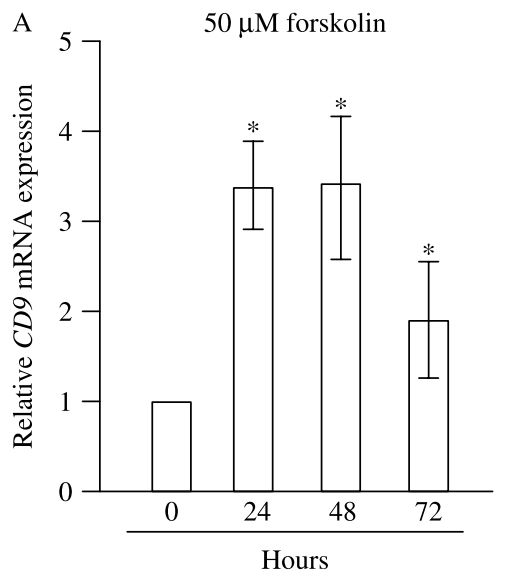

B

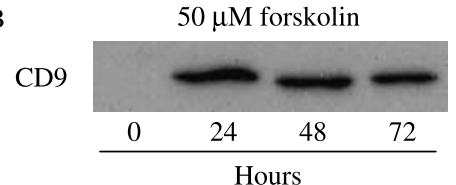

$\mathrm{C}$

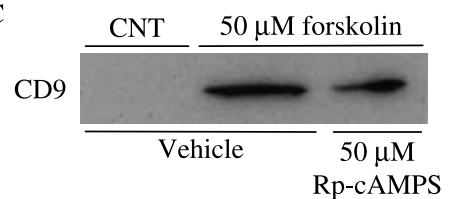

Figure 2 The effects of forskolin on CD9 expression. (A) Real-time PCR analysis of CD9 mRNA in BeWo cells treated with $50 \mu \mathrm{M}$ forskolin for $0,24,48$, or $72 \mathrm{~h}$. Transcript levels are shown as values relative to ACTB mRNA. The data are presented as mean \pm S.E.M. from three independent experiments. ${ }^{*} P<0.05$, compared with $0 \mathrm{~h}$. (B) Western blot analysis of CD9 in BeWo cells treated with $50 \mu \mathrm{M}$ forskolin for 0 , 24,48 , or 72 h. (C) Western blot analysis of CD9 in BeWo cells treated with $50 \mu \mathrm{M}$ forskolin with or without $50 \mu \mathrm{M}$ Rp-cAMPS for $48 \mathrm{~h}$.

increase in transcripts, transient transfection of pSG5-CD9 increased ERVWE1 protein, but not CGB protein (Fig. 3C and D). Next we investigated whether or not CD9 regulated the expression of ERVWE1 mRNA and protein via the CAMP/PKA signaling pathway. Among Rp-cAMPS doses examined, the $50 \mu \mathrm{M}$ dose reduced the increase in ERVWE1 mRNA to levels similar to ERVWE1 expression without the overexpression of the pSG-CD9 construct (Fig. 4A). The $50 \mu \mathrm{M}$ Rp-cAMPS inhibited ERVWE1 protein expression (Fig. 4B). These data indicate that inhibition of the CAMP/PKA signaling pathway prevented the increase in ERVWE1 expression, which had been induced by CD9.

\section{CD9 increases the expression of GCM1, which stimulates ERVWE1 expression}

It has been documented that ERVWE1 gene expression in BeWo cells is enhanced by the transcription factor GCM1 (Yu et al. 2002, Chang et al. 2005). To investigate whether or not CD9 could regulate GCM1, of which mRNA was examined following CD9 transfection into
BeWo cells. Overexpression of pSG5-CD9 increased the abundance of GCM1 mRNA. Similar to ERVWE1 regulation by CD9, increase in GCM1 mRNA was inhibited by the use of Rp-cAMPS (Fig. 5). These observations indicate that the upregulation of GCM1 and ERVWE1 results from the activation of the cAMP/ PKA signaling by the membrane protein CD9.

\section{Discussion}

Evidence has accumulated that ERVWE1 plays an essential role in the formation of binucleate and syncytial trophoblast cells. A factor so far identified in the regulation of ERVWE1 gene transcription is the transcription factor GCM1. In this study, we present the evidence that through CAMP/PKA signaling pathway (Fig. 2), cell membrane protein CD9 could regulate GCM1 and ERVWE1 gene transcription (Fig. 6).

Through increases in ERVWE1 expression, forskolin induces cell-cell fusion in BeWo cells (Mi et al. 2000), resulting in the increase in CGB production (Frendo et al. 2003; Fig. 1). This was also documented by Jameson \& Hollenberg (1993) in which CGB production in
A

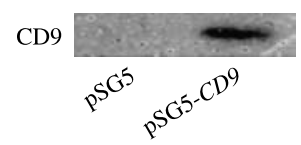

$\mathrm{C}$

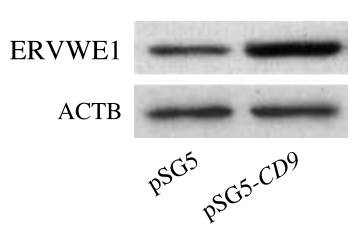

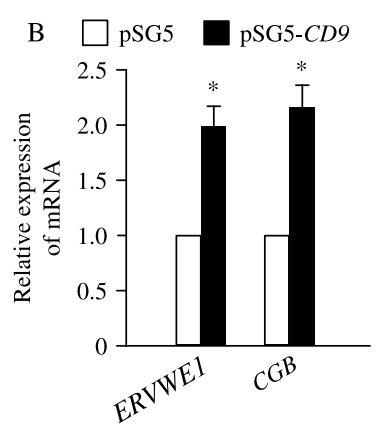

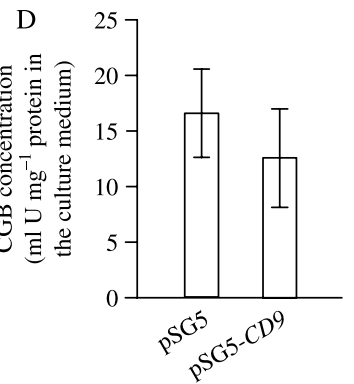

Figure 3 The expression of ERVWE1 and CGB in BeWo cells overexpressing CD9. (A) Western blot analysis of CD9 in BeWo cells transiently transfected with pSG5 (left lane) or pSG5-CD9 (right lane). (B) Abundance of ERVWE1 and CGB mRNA in BeWo cells transiently transfected with pSG5 (white column) or pSG5-CD9 (black column). mRNA levels are shown as values relative to ACTB. The data are presented as mean \pm s.E.M. from three independent experiments. $* P<0.05$, compared with pSG5. (C) Western blot analysis of ERVWE1 in BeWo cells transiently transfected with pSG5 (left lane) or pSG5CD9 (right lane). (D) ELISA of CGB in the medium, from which BeWo cells transiently transfected with pSG5 (left column) or pSG5-CD9 (right column) were cultured. 


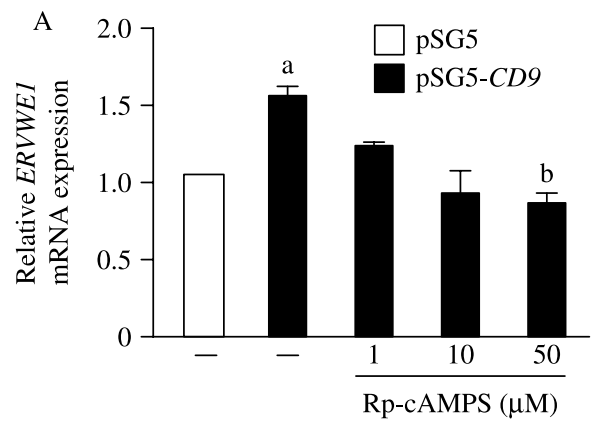

B

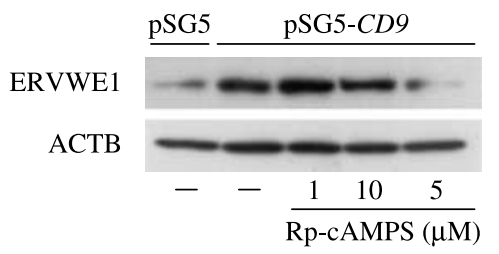

Figure 4 ERVWE1 up-regulation by CD9 via cAMP/PKA signaling pathway. (A) Real-time PCR analysis of ERVWE1 mRNA expression in BeWo cells transiently transfected with pSG5 (white column) or pSG5-CD9 (black columns), which had been treated with or without 1 , 10 , or $50 \mu \mathrm{M}$ Rp-cAMPS, is shown. mRNA levels are shown as values relative to $A C T B$. The data are presented as mean \pm S.E.M. from three independent experiments with duplication. a, $P<0.05$, compared with pSG5. b, $P<0.05$, compared with pSG5-CD9. (B) Western blot analysis of ERVWE1 expression in BeWo cells. The cells were transiently transfected with pSG5 (white column) or pSG5-CD9 (black column), which had been treated with or without 1,10 , or $50 \mu \mathrm{M}$ Rp-cAMPS. A representative gel from three independent western blot analyses is shown.

trophoblast cells results from the cell-cell fusion. CD9 is involved in cell-cell fusion in fertilization (Le Naour et al. 2000) and myogenesis (Tachibana \& Hemler 1999). The previous and present findings suggest that CD9 may also be involved in the regulation of cell-cell fusion and/or ERVWE1 expression. Transient transfection of pSG5-CD9 increased the expression of ERVWE1 mRNA and protein (Fig. 3B and C). In contrast to the treatment with forskolin, $C D 9$ overexpression increased $C G B$ mRNA, but not its protein (Fig. 3B and D). Another human choriocarcinoma cell line, JEG3 cells, does not

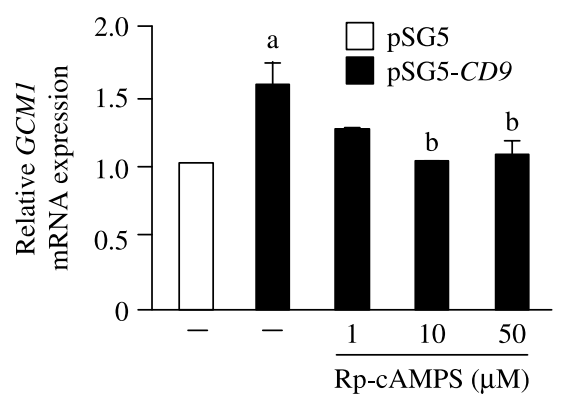

Figure 5 Upregulation of GCM1 mRNA by CD9. The real-time PCR analyses of GCM1 mRNA in BeWo cells transiently transfected with pSG5 (white column) or pSG5-CD9 (black column), which had been treated with or without 1,10 , or $50 \mu \mathrm{M}$ Rp-cAMPS, are shown. a, $P<0.05$, compared with pSG5. b, $P<0.05$, compared with pSG5-CD9.

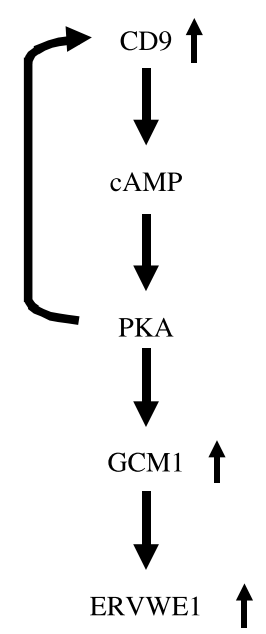

Figure 6 Proposed model of ERVWE1 regulation by CD9 in trophoblast cells. CD9 activates the cAMP/PKA signaling pathway, which increases GCM1 expression, resulting in increases in ERVWE1 expression. The CAMP/PKA signaling pathway also increases CD9 expression, which potentiates CD9-induced expression of ERVWE1.

show any cell-cell fusion regardless of degrees of ERVWE1 expression (data not shown). In addition, whether or not cell-cell fusion is induced by overexpression of SYNCYTIN-A, which is a fusogenic protein of muridae homologous to ERVWE1, is dependent on cell types (Dupressoir et al. 2005). These results suggest that CD9 upregulates ERVWE1 expression, but may not induce cell-cell fusion in BeWo or JEG3 cells.

It was previously shown that ERVWE1 expression is regulated by the CAMP/PKA signaling pathway (Frendo et al. 2003, Chen et al. 2008). In this study, a question of whether or not CD9 regulates ERVWE1 expression via this signaling pathway was examined. The observation in which $50 \mu \mathrm{M}$ of Rp-cAMPS prevented the increase in ERVWE1 expression induced by CD9 (Fig. 4A and B) suggests that CD9 activates PKA, resulting in the increase in ERVWE1 expression. However, at this point, it is unclear how CD9 activates the CAMP/PKA signaling pathway. In fact, CD9 is widely expressed on the plasma membrane of various cell types including trophoblast cells, hematopoietic cells, endothelial cells, and numerous tumor cell lines (Maecker et al. 1997, Berditchevski 2001, Hemler 2003). CD9 is associated with various membrane proteins including $\mathrm{Gi}$ alpha subunit of a heterotrimeric G protein (André et al. 2006, Le Naour et al. 2006), which inhibits adenylate cyclase activity to decrease the production of cAMP (Birnbaumer 2007). CD9 could modulate the activity of the heterotrimeric G protein to regulate the cAMP/PKA signaling pathway. It was demonstrated that GCM1 binds to the upstream region of the ERVWE1 gene and then stimulates $E R V W E 1$ gene transcription in BeWo and JEG3 cells, but not HeLa cells (Yu et al. 2002). In this study, transient transfection of pSG-CD9 increased the expression levels 
of GCM1 mRNA, which was inhibited by Rp-cAMPS (Fig. 5). These results indicate that CD9 may regulate GCM1 and ERVWE1 expression via the cAMP/PKA signaling pathway in a cell-specific manner.

ERVWE1 expression has been implicated in pathological conditions such as preeclampsia (Chen et al. 2006), multiple sclerosis (Antony et al. 2004), and malignant tumor (Strick et al. 2007). Hypoxic condition, which is one of the symptoms observed in preeclampsia (Redman 1991), downregulates ERVWE1 expression (Knerr et al. 2003, Kudo et al. 2003). However, molecular mechanism for the regulation of ERVWE1 expression has not been definitively characterized. In the present study, we report for the first time that the membrane protein CD9 regulates ERVWE1 expression via the cAMP/PKA/GCM1 signaling pathway. Although CD9 interacts with many proteins that mediate signaling between the molecules inside and outside of the cells, or between the molecules on the plasma membrane, it is unclear how CD9 is activated in trophoblast cells. Cytotrophoblast cells express several types of integrins, which play an important role as adhesion molecules in trophoblast cells (Aplin et al. 2009). Though integrins are not typical G protein-coupled proteins, a recent report indicated that integrin beta1, which is associated with CD9 in BeWo cells (Hirano et al. 1999), regulates intracellular cAMP signaling via the activation of $\mathrm{G}$ protein (Alenghat et al. 2009). CD9 could modulate integrin signaling, resulting in activation of cAMP/PKA/ GCM1 signaling. Further experimentation is required to clarify the molecular network involved in the regulation of CD9 and ERVWE1 expression, and of binucleate and syncytial cell formation, a prerequisite for trophoblast development and placental formation.

\section{Materials and Methods}

\section{Cells and cell treatments}

BeWo cells were routinely maintained in Ham's F-12 medium (Sigma-Aldrich) supplemented with $10 \%(\mathrm{v} / \mathrm{v})$ fetal bovine serum (Japan Bioserum, Hiroshima, Japan), $100 \mathrm{U} / \mathrm{ml}$ penicillin, $100 \mu \mathrm{g} / \mathrm{ml}$ streptomycin, $0.25 \mu \mathrm{g} / \mathrm{ml}$ amphotericin B, and $3.7 \mathrm{~g} / \mathrm{l}$ sodium bicarbonate in a humidified atmosphere at $37^{\circ} \mathrm{C}$ in air with $5 \% \mathrm{CO}_{2}$.

In each experiment, 4 or $8 \times 10^{4}$ cells were seeded per well of 12 -well or 6 -well culture plate respectively. When the cells were grown for $2-3$ days to $50 \%$ confluency, culture medium was changed, to which $50 \mu \mathrm{M}$ forskolin were added (Sigma-Aldrich). After 24, 48, or $72 \mathrm{~h}$ culture, the total RNA, protein and culture medium were collected. In some cases, 1, 10 , or $50 \mu \mathrm{M}$ of cAMP inhibitor, Rp-cAMPS (SigmaAldrich), was added $3 \mathrm{~h}$ before the addition of forskolin and the culture continued for $48 \mathrm{~h}$. Doses of forskolin and Rp-cAMPS had been predetermined prior to the initiation of experimentations.

\section{Plasmid construction and transient transfection}

Human CD9 cDNA, a generous gift from Dr Akira Kudo (Tokyo Institute of Technology, Yokohama, Japan), was subcloned into pGEM-T easy vector (Promega). To generate an expression construct, CD9 cDNA was then subcloned into a eukaryotic expression vector pSG5 (Stratagene, La Jolla, CA, USA), resulting in the generation of the pSG5-CD9 expression construct. The expression vector was transfected into BeWo cells using lipofectamine 2000 (Invitrogen) according to the manufacturer's protocol. Transfected BeWo cells with or without forskolin or Rp-cAMPS treatment were cultured for $48 \mathrm{~h}$, and total RNA, protein and/or culture media were then collected.

\section{RNA preparation and quantitative real-time PCR}

Total RNAs were extracted from BeWo cells using ISOGEN (Nippon Gene, Toyama, Japan) according to the manufacturer's instruction. Five microliters of total RNA samples $(250 \mathrm{ng} / \mu \mathrm{l})$ were first RT to cDNA using the cDNA RT kit (Applied Biosystems, Foster City, CA, USA) and oligo (dT) primers in a $20 \mu \mathrm{l}$ reaction volume. The cDNA reaction mixture was diluted 1:5 using Tris-EDTA buffer.

Three microliters of the diluted cDNA reaction mixture were subjected to real-time PCR amplification using 0.5 units of ExTaq HS polymerase (Takara Biomedicals, Tokyo, Japan), $1 \times$ ExTaq HS buffer, $0.5 \mu \mathrm{M}$ of the oligonucleotide primers, SYBR green (SYBR Green I Nucleic Acid Gel stain, Takara Biomedicals) as fluorescence intercalator and Rox reference dye (Invitrogen) in a final volume of $20 \mu \mathrm{l}$, and PCR amplification was carried out in Real-time PCR System (7900HT, Applied Biosystems). Each primer was designed using web-based Primer3 software as follows: ACTB (GenBank accession number: NM_001101.3) (F; 5'-GACTACCTCATGAAGATCCTCPCR-3', R; 5'-CGGATGTCCACGTCGCGTCACACTTC-3'), ERVWE1 (GenBank accession number: NM_014590.3) (F; 5'-ATAACCCATACCTCAAACCTCACC-3', R; 5'-GGCACTAAGAATGAGAGGAAGCA-3'), CGB (GenBank accession number: NM_000737.2) (F; 5'-GCTCACCCCAGCATCCTATC-3', R; 5'-CCTGGAACATCTCCATCCTTG-3'), CD9 (GenBank accession number: NM_001769.2) (F; 5'-GGACGTACTCGAAACCTTCACC-3', R; 5'-GCGGATAGCACAGCACAAGA-3'), GCM1 (GenBank accession number: NM_003643.2) (F; 5'-GATGACAAGGAACCTGACCTAACC-3', R; 5'-AAACAAGAGGCAAGGAGACCAC- $3^{\prime}$ ).

The thermal profile for real-time PCR was at $95^{\circ} \mathrm{C}$ for $10 \mathrm{~min}$, followed by 40 cycles of $95^{\circ} \mathrm{C}$ for $10 \mathrm{~s}, 60^{\circ} \mathrm{C}$ for $20 \mathrm{~s}$, and $72{ }^{\circ} \mathrm{C}$ for $30 \mathrm{~s}$. Average cycle threshold $\left(C_{\mathrm{t}}\right)$ values of each sample were calculated and normalized to $C_{\mathrm{t}}$ values for $A C T B$ using the $2^{-\Delta C_{\mathrm{t}}}$ method. Each run was completed using a melting curve analysis to confirm the specificity of the amplification and the absence of primer dimers (Sakurai et al. 2009).

\section{SDS-PAGE and western blotting analysis}

For the detection of ERVWE1 and ACTB, whole-cells were lysed using RIPA buffer $(50 \mathrm{mM}$ Tris- $\mathrm{HCl}, 150 \mathrm{mM} \mathrm{NaCl}, 1 \mathrm{mM}$ EDTA, $1 \mathrm{mM} \mathrm{NaVO}_{4}, 50 \mathrm{mM} \mathrm{NaF}, 0.1 \%$ (w/v) SDS, and $1 \%$ $(\mathrm{v} / \mathrm{v})$ Triton $\mathrm{X}-100)$ containing $1 \mathrm{mM}$ dithiothreitol, $2 \mathrm{mM}$ 
phenylmethylsulfonyl fluoride, $2 \mu \mathrm{g} / \mathrm{ml}$ aprotinin, $5 \mu \mathrm{g} / \mathrm{ml}$ leupeptin, and $1 \mu \mathrm{g} / \mathrm{ml}$ pepstatin A. Protein concentration of each sample was determined using the protein assay kit II (BioRad). Protein samples $(10 \mu \mathrm{g})$ were boiled for $5 \mathrm{~min}$ in the SDS sample buffer, which were then applied onto each lane of $12.5 \%$ SDS-PAGE gel under reducing conditions. For the detection of a membrane protein CD9, $5 \times 10^{4}$ cells were lysed in $100 \mu \mathrm{l}$ sample buffer $(20 \mathrm{mM}$ Tris- $\mathrm{HCl} \mathrm{pH} 8.6,1 \%(\mathrm{w} / \mathrm{v})$ SDS, $20 \%(\mathrm{v} / \mathrm{v})$ glycerol and bromophenol blue), containing $10 \times$ SDS versus ERVWE1 detection RIPA buffer. Ten microliters of lysed sample were then applied to each lane of $12.5 \%$ SDSPAGE gel under reducing conditions.

For western blot analysis, the proteins in the gel were transferred onto nitrocellulose membranes (Immobilon, Millipore, Bedford, MA, USA). The membranes were blocked in $5 \%(\mathrm{w} / \mathrm{v})$ skim milk dissolved with Tris-buffered saline containing $0.5 \%(\mathrm{v} / \mathrm{v})$ Tween (TBST) at room temperature for $1 \mathrm{~h}$ and then incubated with a rabbit polyclonal antibody to ERVWE1 (1:10 000, H-280, Santa Cruz, CA, USA), a mouse $M A B$ to $A C T B(1: 5000, F 3022$, Sigma-Aldrich) or a mouse MAB to CD9 (1:10 000, K41, BMA Biomedicals AG, Augst, Switzerland) at $4{ }^{\circ} \mathrm{C}$ for $12 \mathrm{~h}$. After incubation, membranes were washed three times in TBST, and incubated in donkey anti-rabbit or anti-mouse IgG-conjugated with HRPs (GE Healthcare Bio-Sciences AB, Bjorkgatan, Sweden) at room temperature for $1 \mathrm{~h}$. After washing the membrane three times in TBST, the bands were identified using ECL plus western blotting detection reagents (GE Healthcare UK Ltd, Buckinghamshire, UK).

\section{CGB measurement in culture medium}

The collected medium was centrifuged at $400 \mathrm{~g}$ at $4{ }^{\circ} \mathrm{C}$ for $3 \mathrm{~min}$ to remove cellular debris. After total protein concentrations were adjusted to $1 \mathrm{mg} / \mathrm{ml}$ using the culture medium, CGB concentration of each medium was determined using the ELISA kit (Immuno-Biological Laboratories, Hamburg, Germany), which is based on the sandwich principle and specifically detects CGB according to the manufacturer's instruction.

\section{Statistical analysis}

The concentration of CGB protein, the expression level of ERVWE1 protein and CD9 mRNA after the addition of forskolin, and the expression level of ERVWE1 and GCM1 RNAs after the addition of Rp-cAMPS were analyzed using oneway ANOVA, followed by Tukey-Kramer test. The effect of transient transfection of pSG5-CD9 on the expressions of CGB protein or ERVWE1 and $C G B$ mRNAs was analyzed by $F$ test followed by Student's $t$-test. $P$ value $<0.05$ was considered statistically significant.

\section{Declaration of interest}

The authors declare that there is no conflict of interest that could be perceived as prejudicing the impartiality of the research reported.

\section{Funding}

This research was supported by Grants-in-aid for Scientific Research (18108004) from the Japan Society for the Promotion of Science and by the Program for Promotion of Basic Research Activities for Innovative Bioscience (BRAIN).

\section{Acknowledgements}

The authors thank Dr Akira Kudo, Tokyo Institute of Technology, Yokohama, Japan, for generously proving CD9 cDNA.

\section{References}

Alenghat FJ, Tytell JD, Thodeti CK, Derrien A \& Ingber DE 2009 Mechanical control of cAMP signaling through integrins is mediated by the heterotrimeric Galphas protein. Journal of Cellular Biochemistry 106 529-538.

André M, Le Caer JP, Greco C, Planchon S, El Nemer W, Boucheix C, Rubinstein E, Chamot-Rooke J \& Le Naour F 2006 Proteomic analysis of the tetraspanin web using LC-ESI-MS/MS and MALDI-FTICR-MS. Proteomics 6 1437-1449.

Antony JM, van Marle G, Opii W, Butterfield DA, Mallet F, Yong VW, Wallace JL, Deacon RM, Warren K \& Power C 2004 Human endogenous retrovirus glycoprotein-mediated induction of redox reactants causes oligodendrocyte death and demyelination. Nature Neuroscience 7 1088-1095.

Aplin JD 1991 Implantation, trophoblast differentiation and haemochorial placentation: mechanistic evidence in vivo and in vitro. Journal of Cell Science 99 681-692.

Aplin JD, Jones CJ \& Harris LK 2009 Adhesion molecules in human trophoblast - a review. I. Villous trophoblast. Placenta 30 293-298.

Benirschke K \& Kaufmann P 2001 Pathology of the Human Placenta, edn 4, p 49. New York: Springer-Verlag.

Berditchevski F 2001 Complexes of tetraspanins with integrins: more than meets the eye. Journal of Cell Science 114 4143-4151.

Birnbaumer L 2007 Expansion of signal transduction by $\mathrm{G}$ proteins. The second 15 years or so: from 3 to 16 alpha subunits plus betagamma dimers. Biochimica et Biophysica Acta 1768 772-793.

Blaise S, de Parseval N, Bénit L \& Heidmann T 2003 Genomewide screening for fusogenic human endogenous retrovirus envelopes identifies syncytin-2, a gene conserved on primate evolution. PNAS 100 13013-130138.

Blond JL, Lavillette D, Cheynet V, Bouton O, Oriol G, Chapel-Fernandes S, Mandrand B, Mallet F \& Cosset FL 2000 An envelope glycoprotein of the human endogenous retrovirus HERV-W is expressed in the human placenta and fuses cells expressing the type D mammalian retrovirus receptor. Journal of Virology 74 3321-3329.

Boucheix C \& Rubinstein E 2001 Tetraspanins. Cellular and Molecular Life Sciences 58 1189-1205.

Chang CW, Chuang HC, Yu C, Yao TP \& Chen H 2005 Stimulation of GCMa transcriptional activity by cyclic AMP/protein kinase A signaling is attributed to CBP-mediated acetylation of GCMa. Molecular and Cellular Biology 25 8401-8414.

Chen CP, Wang KG, Chen CY, Yu C, Chuang HC \& Chen H 2006 Altered placental syncytin and its receptor ASCT2 expression in placental development and preeclampsia. British Journal of Obstetrics and Gynaecology 113 152-158.

Chen CP, Chen LF, Yang SR, Chen CY, Ko CC, Chang GD \& Chen H 2008 Functional characterization of the human placental fusogenic membrane protein syncytin-2. Biology of Reproduction 79 815-823.

Dupressoir A, Marceau G, Vernochet C, Bénit L, Kanellopoulos C, Sapin V \& Heidmann T 2005 Syncytin-A and syncytin-B, two fusogenic placentaspecific murine envelope genes of retroviral origin conserved in Muridae. PNAS 102 725-730.

Feinman MA, Kliman HJ, Caltabiano S \& Strauss JF III 1986 8-Bromo-3', 5'adenosine monophosphate stimulates the endocrine activity of human cytotrophoblasts in culture. Journal of Clinical Endocrinology and Metabolism 63 1211-1217. 
Ferretti C, Bruni L, Dangles-Marie V, Pecking AP \& Bellet D 2007 Molecular circuits shared by placental and cancer cells, and their implications in the proliferative, invasive and migratory capacities of trophoblasts. Human Reproduction Update 13 121-141.

Frendo JL, Olivier D, Cheynet V, Blond JL, Bouton O, Vidaud M, Rabreau M, Evain-Brion D \& Mallet F 2003 Direct involvement of HERV-W Env glycoprotein in human trophoblast cell fusion and differentiation. Molecular Biology of the Cell 23 3566-3574.

Ha CT, Waterhouse R, Wessells J, Wu JA \& Dveksler GS 2005 Binding of pregnancy-specific glycoprotein 17 to CD9 on macrophages induces secretion of IL-10, IL-6, PGE2, and TGF- $\beta 1$. Journal of Leukocyte Biology 77 948-957.

Hemler ME 2003 Tetraspanin proteins mediate cellular penetration, invasion, and fusion events and define a novel type of membrane microdomain. Annual Review of Cell and Developmental Biology 19 397-422.

Hirano T, Higuchi T, Katsuragawa H, Inoue T, Kataoka N, Park KR, Ueda M, Maeda M, Fujiwara H \& Fujii S 1999 CD9 is involved in invasion of human trophoblast-like choriocarcinoma cell line, BeWo cells. Molecular Human Reproduction 5 168-174.

Jameson JL \& Hollenberg AN 1993 Regulation of chorionic gonadotropin gene expression. Endocrine Reviews 14 203-221.

Knerr I, Weigel C, Linnemann K, Dötsch J, Meissner U, Fusch C \& Rascher W 2003 Transcriptional effects of hypoxia on fusiogenic syncytin and its receptor ASCT2 in human cytotrophoblast BeWo cells and in ex vivo perfused placental cotyledons. American Journal of Obstetrics and Gynecology 189 583-588.

Kudo Y, Boyd CA, Sargent IL \& Redman CW 2003 Hypoxia alters expression and function of syncytin and its receptor during trophoblast cell fusion of human placental BeWo cells: implications for impaired trophoblast syncytialisation in preeclampsia. Biochimica et Biophysica Acta 1638 63-71.

Maecker HT, Todd SC \& Levy S 1997 The tetraspanin superfamily: molecular facilitators. FASEB Journal 11 428-442.

Malassiné A, Blaise S, Handschuh K, Lalucque H, Dupressoir A, EvainBrion D \& Heidmann T 2007 Expression of the fusogenic HERV-FRD Env glycoprotein (syncytin-2) in human placenta is restricted to villous cytotrophoblastic cells. Placenta 28 185-191.
Midgley AR, Pierce GB Jr, Deneau GA \& Gosling JR 1963 Morphogenesis of syncytiotrophoblast in vivo: an autoradiographic demonstration. Science 141 349-350.

Mi S, Lee X, Li X, Veldman GM, Finnerty H, Racie L, LaVallie E, Tang XY, Edouard P, Howes S et al. 2000 Syncytin is a captive retroviral envelope protein involved in human placental morphogenesis. Nature 403 785-789.

Le Naour F, Rubinstein E, Jasmin C, Prenant M \& Boucheix C 2000 Severely reduced female fertility in CD9-deficient mice. Science 287 319-321.

Le Naour F, André M, Greco C, Billard M, Sordat B, Emile JF, Lanza F, Boucheix C \& Rubinstein E 2006 Profiling of the tetraspanin web of human colon cancer cells. Molecular \& Cellular Proteomics 5 845-857.

Redman CW 1991 Current topic: preeclampsia and the placenta. Placenta 12 301-308.

Sakurai T, Sakamoto A, Muroi Y, Bai H, Nagaoka K, Tamura K, Takahashi T, Hashizume K, Sakatani M, Takahashi M et al. 2009 Induction of endogenous tau interferon gene transcription by CDX2 and high acetylation in bovine non-trophoblast cells. Biology of Reproduction 80 1223-1231.

Strick R, Ackermann S, Langbein M, Swiatek J, Schubert SW, Hashemolhosseini S, Koscheck T, Fasching PA, Schild RL, Beckmann MW et al. 2007 Proliferation and cell-cell fusion of endometrial carcinoma are induced by the human endogenous retroviral syncytin- 1 and regulated by TGF- $\beta$. Journal of Molecular Medicine $\mathbf{8 5}$ 23-38.

Tachibana I \& Hemler ME 1999 Role of transmembrane 4 superfamily (TM4SF) proteins CD9 and CD81 in muscle cell fusion and myotube maintenance. Journal of Cell Biology 146 893-904.

Yu C, Shen K, Lin M, Chen P, Lin C, Chang GD \& Chen H 2002 GCMa regulates the syncytin-mediated trophoblastic fusion. Journal of Biological Chemistry 277 50062-50068.

Received 4 March 2009

First decision 6 April 2009

Revised manuscript received 18 June 2009

Accepted 19 August 2009 